\title{
Cosmological observations to shed light on possible variations
}

\author{
M. Wendt ${ }^{1}$, D. Reimers ${ }^{1}$ and P. Molaro ${ }^{2}$ \\ ${ }^{1}$ Hamburger Sternwarte, Universität Hamburg, Gojenbergsweg 112, 21029 Hamburg, Germany \\ ${ }^{2}$ INAF -Osservatorio Astronomico di Trieste, Via Tiepolo 11, I-34143 Trieste, Italy
}

Cosmology contributes a good deal to the investigation of variation of fundamental physical constants. High resolution data is available and allows for detailed analysis over cosmological distances and a multitude of methods were developed. The raised demand for precision requires a deep understanding of the limiting errors involved. The achievable accuracy is under debate and current observing proposals max out the capabilities of today's technology. The question for self-consistency in data analysis and effective techniques to handle unknown systematic errors is of increasing importance. This work is motivated by numerous findings of different groups that partially are in disagreement witch each other. A large part of these discrepancies reflects the different methods of handling systematic errors. Evidently systematics are not yet under control or fully understood. We try to emphasize the importance to take these errors, namely calibration issues, into account and put forward some measures adapted to the problem. Alternative approaches for some of the steps involved are introduced.

Two independent observations of the Quasar Q0347-383 are compared to illustrate the problem of apparent precision when relying on error estimates of fitting algorithms alone. We show that the confidence to have accomplished the best numerical fit to a given data set is not very strongly correlated to the level of accuracy reached in terms of the real physical parameters. Numerous fitting algorithms used today are tested in detail for their ability to give best results for certain data. Recent works showed a trend to convert the level of statistical robustness of a fit into the resulting total error of the analysis. This approach lead to seemingly positive detections of variation in the past due to underestimated systematic errors in the reduced data itself. This work offers a test to verify the reproducibility of an analysis and it also emphasizes the plain need for better wavelength calibration in the future. Some of the approaches presented are suitable to handle absorption spectra up to that point.

In addition some procedures to deal with uncertainties in the calculated sensitivity coefficients for molecular hydrogen are highlighted. Unlike the transition frequencies for the Lyman and Werner bands, the calculated sensitivity coefficients cannot be verified in laboratories. Complex non-adiabatic effects have to be taken into account. At the present state the influence of the estimated errors in the sensitivity coefficients are negligible. However, they influence the error estimate of the analysis in principle. An issue of increasing importance for future data analysis with significantly lower uncertainties in wavelength calibration. 